\title{
Fatal Rectal Bleeding after Prostate Biopsy
}

Min Jung Kim¹, Jae-Hong Park², Byung $\mathrm{Ha} \mathrm{Choi}^{1}$, Nak-Eun Chung ${ }^{1}$

${ }^{1}$ Division of Forensic Medicine, National Forensic Service, Seoul, Korea

${ }^{2}$ Division of Forensic Medicine, the Central District of National

Forensic Service, Daejeon, Korea

Received : April 17, 2012

Accepted : May 10, 2012

Corresponding Author :

Min Jung Kim

Division of Forensic Medicine, National Forensic Service, 139 Jiyangno, Sinwol-dong, Yangcheon-gu, Seoul 158-707, Korea

TEL : (822) 2600-4613

FAX : (822) 2600-4828

E-mail : lillu79@korea.kr
Transrectal ultrasound (TRUS)-guided biopsy of the prostate is a common outpatient procedure for diagnosing prostatic cancer. It is a relatively noninvasive, safe, and effective method, as the majority of post-biopsy complications are mild and self-limiting. Fatal complications including infection or massive hemorrhage are rare, but can be life threatening. We herein present an autopsy case of fatal rectal bleeding after prostate biopsy and an analysis of similar cases.

Key words : prostate biopsy, complications, bleeding, rectal, fatal

\section{Introduction}

Transrectal ultrasound (TRUS)-guided biopsy of the prostate is a gold-standard for diagnosing the prostatic cancer. It is a relatively less invasive technique and known to be fast, safe, and effective method, because post biopsy complications including gross hematuria, rectal bleeding, hematospermia, fever and chill are mostly self-limiting and the rate of major complications are low.

Rare major complications include acute prostatitis, acute urinary retention, epididymitis, severe hematuria, sepsis, abscess formation, urinary tract infection, tumor tracking, vasovagal syncope, and significant rectal bleeding. ${ }^{1,21}$ A nation-wide survey in
Japan reported that the $5.9 \%$ of 212,065 men undergone rectal bleeding after the procedure. ${ }^{3)}$ And a large prospective series of prostatic biopsies showed the $8.2 \%$ of patients reported moderate to severe rectal bleeding after the biopsy. ${ }^{4}$ Chiang et al. ${ }^{21}$ reported that the rate of rectal bleeding needed hospitalization is $0.2 \%$. The major ones occur very rarely but if they occur, it could be life-threatening.

We herein present an autopsy case of fatal rectal bleeding after prostate biopsy to share how we handle this rare case and review the reported similar cases by analyzing the papers.

\section{Case Report}

A 54-year-old man with the history of hypertension 
for 20 years and chronic prostatitis for 5 years was found to have prostate-specific antigen (PSA) at 4.44 $\mathrm{ng} / \mathrm{mL}$ (normal < $3.5 \mathrm{ng} / \mathrm{dL}$ ) during a health screening. He underwent double sextant TRUS-guided prostate multiple biopsy (12 cores) with an 18-gauge needle after local anesthesia with lidocaine. The prostate volume measured $66.2 \mathrm{cc}$ in total, $24.7 \mathrm{cc}$ in transitional zone. After 30 minutes from the procedure, he complained of cold sweating, nausea, vomiting, and dizziness. He noticed rectal bleeding and thereafter he continued to pass a large volume of bright red blood through the rectum. But two hours later, the doctor decided his discharge after selfvoiding. He complained of that nausea, dizziness, and vomiting and rectal bleeding is ongoing while he is going home with his wife by car. Six hours later, he revisited the emergency room for vomiting and persisting rectal bleeding. Manual compression and conservative therapy failed to stop the bleeding. Red blood cell units were transfused and endoscopic consultation was requested. Emergency sigmoidoscopy showed the hematoma of just above the anal

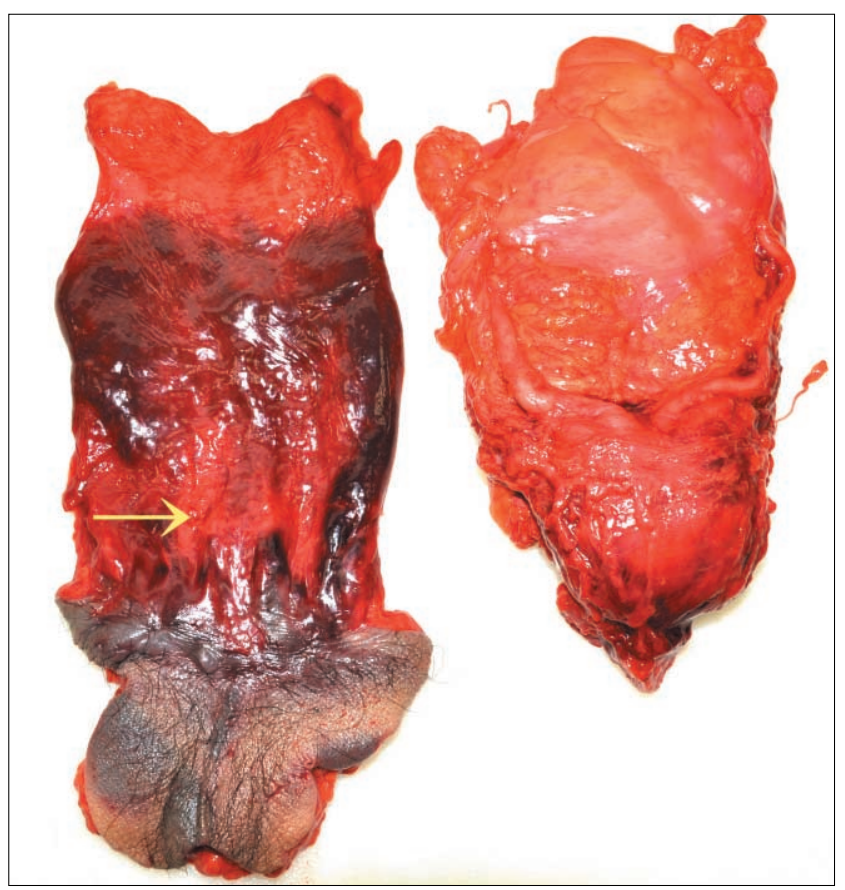

Fig. 1. The rectal mucosa just above anal verge shows hemorrhage and a mucosal perforation (arrow). Note the left posterior side of prostate under the perforation has more rich vascular bed than the right posterior side of it. verge, but mucosal region was not seen because of the blood filled in colonic lumen block the view. And 13 hours later, He died and an autopsy was performed for medicolegal investigation.

The deceased revealed multiple bruise at perineal region, especially diffuse submucosal hemorrhage and swelling of anal and perianal area. The large bowel is expanded and filled with fresh blood. Anus, rectum, prostate, urinary bladder were removed totally. Rectal mucosa showed submucosal bleeding at just above the anal verge and the mucosal perforation at anterior wall, $3 \mathrm{~cm}$ above from the anal verge, over the left side of the underlying prostate. The left posterior side of the prostate is supplied by more rich vascular bed that might be consists of branches of the inferior vesicular arteries than right posterior side of it (Fig. 1). The prostate revealed multiple small hemorrhagic foci and chronic prostatitis was noted on microscopy. The heart was $436 \mathrm{gm}$ in weights and showed severe coronary atherosclerosis and ventricular patchy fibrosis. And acute ischemic necrosis of ventricle was identified microscopically. The liver revealed severe fatty changes. No evidence of remarkable disease or injury was identified in the lungs, kidneys, and spleen. No noteworthy drugs, poisonous agents or chemicals were detected in the blood and the gastric contents excepting atropine and lidocaine was used during the treatment.

The medical records and autopsy findings lead to concluding the cause of death to be a hypovolemic shock due to rectal hemorrhage after the TRUS-guided multiple prostate biopsies.

\section{Discussion}

The widespread use of PSA as a tool for prostate cancer screening, High sensitivity and low specificity of PSA led to increase of prostate biopsy. TRUS-guided multiple prostate biopsy is usually performed in an outpatient setting because the procedure is generally considered fast and safe. However, several prospective and retrospective studies have shown that minor complications, usually mild and self-limiting, are not 
infrequent, and the major ones are rare, but could be fatal. Newer biopsy protocols suggest an increase in sampling numbers for avoiding missing the existing cancers. ${ }^{5}$ There is an insist that the increasing of the number of core samples lead to no increase in severity of side effects, ${ }^{6}{ }^{6}$ but the total incidence of rectal bleeding is listed as $1.3-58.6 \%$, with a statistically significant positive correlation to the number of core samples obtained in another study.1 Although the most of bleeding complications are slight and not needed a further therapeutic intervention, but massive rectal bleedings could occur and be life-threatening.

Risk factors of rectal bleeding have not yet been specified. According to Gonen, a large hypoechoic hypervascular area of the prostate might be an risk factor for post-biopsy rectal bleeding. ${ }^{71}$ Patients with rich vascular bed surrounding rectum and prostate that consists of branches of the inferior vesicular arteries, the middle and inferior rectal arteries, and dense submucosal venous plexus could be susceptible to rectal bleeding. ${ }^{8}$ Patients with larger prostate size were noted to have higher risk of developing transient acute prostatitis and acute urinary retention after prostate biopsy, but they were not associated with rectal hemorrhage. And age, prophylactic antibiotics, underlying diseases, and antiplatelet/anticoagulant usage were not associated with major complications after prostate biopsy. ${ }^{21}$

We found 9 publications that describe post prostate biopsy massive rectal bleeding by an extensive research of Medline using the key words rectal bleeding and TRUS-guided prostate biopsy. Nineteen life-threatening rectal bleeding cases are introduced. The known patients' age is between 57 and 81 years. The core numbers of needle biopsies are between 6 and 18 cores. None of them died of bleeding. All the bleeding are successfully controlled by endoscopic hemostasis with rectal tamponade by means of a condom filled with fluid in one case or endoscopic intervention with injection of $1 \%$ athexysclerol, epinephrine, polidocanol, and adrenalin, thermocoagulation, band ligation, and endoclips in the others. ${ }^{2,}$. ${ }^{14}$ Evaluation by urgent endoscopy and performing bleeding control allows accurate diagnosis and appropriate treatments.

The high risk patients are evaluated by interview about important underlying disease, antiplatelet/ anticoagulant usage, etc. and laboratory examination and they should undergo the biopsy as an inpatient. Informed to the patient about the exact procedure and possible complications by interviewing with medical team or leaflets for TRUS-guided prostate biopsies would be helpful for patients to understand the procedure and communicate more effectively with health professionals. The most important way to minimize the risks of a major rectal bleeding and death is post biopsy close observation, early detection of the complication, and appropriate bleeding control by endoscopic interventions.

\section{References}

1. Raaijmakers R, Kirkels WJ, Roobol MJ, Wildhagen MF, Schrder FH. Complication rates and risk factors of 5802 transrectal ultrasound-guided sextant biopsies of the prostate within a population-based screening program. Urology 2002;60:826-30.

2. Chiang IN, Chang SJ, Pu YS, Huang KH, Yu HJ, Huang CY. Major complications and associated risk factors of transrectal ultrasound guided prostate needle biopsy: a retrospective study of 1875 cases in taiwan. J Formos Med Assoc 2007;106:929-34.

3. Kakehi Y, Naito S. Complication rates of ultrasound-guided prostate biopsy: a nation-wide survey in Japan. Int J Urol 2008;15:319-21.

4. Rodriguez LV, Terris MK. Risks and complications of transrectal ultrasound guided prostate needle biopsy: a prospective study and review of the literature. J Urol 1998;160:2115-20.

5. Naughton CK, Miller DC, Mager DE, Ornstein DK, Catalona WJ. A prospective randomized trial comparing 6 versus 12 prostate biopsy cores: impact on cancer detection. J Urol 2000;164:388-92.

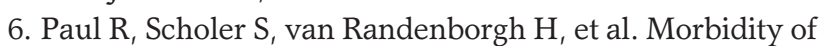
prostatic biopsy for different biopsy strategies: is there a relation to core number and sampling region? Eur Urol 2004; $45: 450-5$

7. Gonen M, Resim S. Simplified treatment of massive rectal bleeding following prostate needle biopsy. Int J Urol 2004; $11: 570-2$.

8. Katsinelos P, Kountouras J, Dimitriadis G, et al. Endoclipping treatment of life-threatening rectal bleeding after prostate biopsy. World J Gastroenterol 2009;15:1130- 
3.

9. Ustündağ Y, Yeşilli C, Aydemir S, Savranlar A, Yazicioglu $\mathrm{K}$. A life-threatening hematochesia after transrectal ultrasound-guided prostate needle biopsy in a prostate cancer case presenting with lymphedema. Int Urol Nephrol 2004;36:397-400.

10. Strate LL, O'Leary MP, Carr-Locke DL. Endoscopic treatment of massive rectal bleeding following prostate needle biopsy. Endoscopy 2001;33:981-4.

11. Brullet E, Guevara MC, Campo R, et al. Massive rectal bleeding following transrectal ultrasound-guided prostate biopsy. Endoscopy 2000;32:792-5.

12. Geraci G, Sciume C, Pisello F, Facella T, Modica G. Severe rectal bleeding after transrectal US-guided prostate biopsy. Case report. G Chir 2006;27:321-3.

13. Braun KP, May M, Helke C, Hoschke B, Ernst H. Endoscopic therapy of a massive rectal bleeding after prostate biopsy. Int Urol Nephrol 2007;39:1125-9.

14. Kinney TP, Kozarek RA, Ylvisaker JT, Gluck M, Jiranek GC, Weissman R. Endoscopic evaluation and treatment of rectal hemorrhage after prostate biopsy. Gastrointest Endosc 2001;53:117-9. 